\title{
Formal Models of Awareness
}

\author{
Thomas Ågotnes · Natasha Alechina
}

Published online: 17 June 2014

(C) Springer Science+Business Media Dordrecht 2014

This special issue on Formal Models of Awareness contains five papers that concentrate on different approaches to the notion of awareness. They include syntactic and semantic approaches to modelling awareness (awareness of formulas as opposed to awareness of semantic objects such as sets of possoble worlds) and an alternative view from the multi-agent systems perspective where awareness is identified as the ability to perceive and understand actions of other agents.

Velázquez-Quesada studies a logic of plausibility acknowledgement models used to interpret the notions of implicit and explicit beliefs. The models use the notion of a formula being acknowledged as true that is similar to syntactic awareness in that the set of formulas acknowledged as true by an agent is not assumed to be closed under logical consequence. However it has a different meaning (of not just being aware of the formula, but being aware that the formula is is true). A dynamic epistemic logic over such models is considered, and it is shown how to model belief revision and inferences performed by agents.

The paper by Soler-Toscano and Velázquez-Quesada belongs to the same tradition of considering explicit information or awareness of non-omniscient reasoners. It focusses on the problem of abductive reasoning for agents whose information is not closed under consequence and who may not have complete reasoning abilities. It generalises the abductive problem for non-omnisient reasoners as finding a hypothesis which, under the agent's inferential abilities and explicit information, would entail a given observation. Furthermore, it formalises abductive reasoning by non-omniscient

\footnotetext{
T. Ågotnes $(\varangle)$

University of Bergen, Bergen, Norway

e-mail: thomas.agotnes@infomedia.uib.no

N. Alechina

University of Nottingham, Nottingham, UK

e-mail: natasha.alechina@nottingham.ac.uk
} 
agents in a version of dynamic epistemic logic with operations on models that involve changing plausibility relations (upgrading) and adding formulas to the information the agent is explicitly aware of (uncovering).

Van Ditmarsch and French develop several logics that combine knowledge and awareness of propositional variables and other agents, and model change of awareness (becoming aware and forgetting). Their work belongs to the semantic treatment of awareness: within the limits of their awareness, the agents are fully rational, and their explicit knowledge is closed under consequence.

The paper by Ågotnes and Alechina in a way combines the syntactic and semantic approaches in that it considers awareness sets as sets of formulas, but under the assumption that they are generated by primitive propositions. The paper considers a logic that can be used to reason about unawareness. The language can, for example, express that an agent knows that he is not aware of everything, and that there are things another agent is aware of that he is not.

The paper by Saunier, Balbo and Pinson studies awareness of artificial agents in a multi-agent system. By awareness, the authors mean the ability to perceive and understand the activities of others. They focus on awareness of other agents' communication. Their work addresses the technical challenge of enabling agents to become aware of communication between other agents and 'overhear' it. The authors solve it by proposing a model of an 'active' environment that mediates agents' interactions. They also propose an integration of awareness (adding a role of listener) into communication protocols. The paper proposes a concrete mechanism for the agents in a multi-agent system to be able to perceive (become aware of) communication of other agents. 